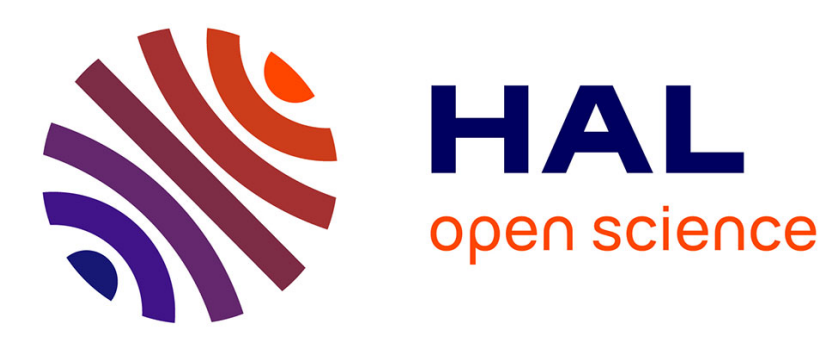

\title{
Influence des contraintes de traction sur les propriétés magnétiques de rubans amorphes MG 2605 Co
}

\author{
A. Nabais, J.C. Faugières, J.F. Rialland, Raymonde Bonnefille
}

\section{To cite this version:}

A. Nabais, J.C. Faugières, J.F. Rialland, Raymonde Bonnefille. Influence des contraintes de traction sur les propriétés magnétiques de rubans amorphes MG 2605 Co. Revue de Physique Appliquée, 1984, 19 (1), pp.1-5. 10.1051/rphysap:019840019010100 . jpa-00245152

\section{HAL Id: jpa-00245152 https://hal.science/jpa-00245152}

Submitted on 1 Jan 1984

HAL is a multi-disciplinary open access archive for the deposit and dissemination of scientific research documents, whether they are published or not. The documents may come from teaching and research institutions in France or abroad, or from public or private research centers.
L'archive ouverte pluridisciplinaire HAL, est destinée au dépôt et à la diffusion de documents scientifiques de niveau recherche, publiés ou non, émanant des établissements d'enseignement et de recherche français ou étrangers, des laboratoires publics ou privés. 
REVUE DE PHYSIQUE APPLIQUÉE

Revue Phys. Appl. 19 (1984) 1-5

JANVIER 1984, PAGE 1

Classification

Physics Abstracts

$75.50 \mathrm{~K}$

\title{
Influence des contraintes de traction sur les propriétés magnétiques de rubans amorphes MG 2605 Co
}

\author{
A. Nabais, J. C. Faugières, J. F. Rialland et R. Bonnefille \\ Conservatoire National des Arts et Métiers, Laboratoire d'Electricité Industrielle, \\ 292, rue Saint-Martin, 75141 Paris Cedex 03, France
}

(Reçu le 6 juin 1983, révisé le 12 septembre, accepté le 17 octobre 1983)

\begin{abstract}
Résumé. - Les propriétés magnétiques de rubans ferromagnétiques amorphes MG $2605 \mathrm{Co}$, bruts de trempe, sont déterminées, en fonction de contraintes de traction allant jusqu'à $75 \mathrm{daN} . \mathrm{mm}^{-2}$, dans un domaine de fréquence compris entre $50 \mathrm{~Hz}$ et $50 \mathrm{kHz}$. Les résultats montrent que la perméabilité d'impédance augmente avec la contrainte $\sigma$. En ce qui concerne les pertes, le comportement diffère à basse et haute fréquence. Cela traduit une évolution différente des pertes par hystérésis et courants de Foucault avec la contrainte exercée. Dans le domaine des faibles contraintes $\left(\sigma<10 \mathrm{daN} . \mathrm{mm}^{-2}\right)$, les observations rendent compte d'une anisotropie uniaxiale induite par l'effort de traction, analogue à celle créée lors d'un recuit sous champ magnétique.
\end{abstract}

\begin{abstract}
The magnetic properties of as-cast amorphous ribbon alloys MG 2605 Co are settled over a frequency range $50 \mathrm{~Hz}-50 \mathrm{kHz}$ and with applied tensile stresses up to $75 \mathrm{daN}$. $\mathrm{mm}^{-2}$. The results show that the AC permeability increases with the applied stress $\sigma$. The influence of tensile load on the hysteresis loss and the eddy current loss is quite different according to the frequency. So, the results exhibit a reduction in total losses at the lower frequencies produced by the tensile stress and an increase at the higher frequencies. For $\sigma<10 \mathrm{daN} . \mathrm{mm}^{-2}$, it is concluded that applied tensile stress induce magnetic anisotropy, similar to anisotropy achieved by field annealing.
\end{abstract}

\section{Introduction.}

Les matériaux ferromagnétiques amorphes se distinguent par de faibles pertes électromagnétiques et, à l'état non recuit, par une grande résistance à la traction. Aussi, l'utilisation de ces matériaux dans la réalisation de machines électriques tournantes doit conduire à un accroissement sensible de leurs performances, en permettant un fonctionnement à fréquence et vitesse de rotation élevées. Cette dernière condition a comme conséquence de soumettre le matériau constituant le rotor de la machine à de fortes contraintes. Les ferromagnétiques amorphes riches en $\mathbf{F e}$ ayant un coefficient de magnétostriction important [1-3], il est nécessaire de caractériser l'évolution de leurs propriétés magnétiques en fonction de la contrainte exercée.

Le traitement thermique du matériau, sous un champ magnétique appliqué selon l'axe du ruban, entraîne, en créant une anisotropie uniaxiale, une augmentation de la perméabilité et du rapport entre l'induction rémanente et l'induction à saturation [4-6]. Une même anisotropie peut être obtenue à l'aide d'un recuit en présence d'une contrainte longitudinale [7-8].
Du point de vue de l'utilisateur, ces traitements ont l'inconvénient d'augmenter les pertes à haute fréquence et de fragiliser le matériau. Le premier effet est lié à l'accroissement des pertes par courants de Foucault $\mathrm{du}$ fait de la structure magnétique induite lors du traitement. D'autres recuits (sous champ oblique en particulier) permettent de réduire ces pertes mais également la perméabilité du matériau [9-10]. La fragilisation des échantillons après traitement thermique correspond à un phénomène encore mal compris.

Pour l'application envisagée, les matériaux bruts de trempe semblent, pour l'instant, les mieux adaptés. Par ailleurs les rubans amorphes MG 2605 Co, fabriqués par Allied Chemical Co, sont ceux qui présentent l'induction à saturation la plus élevée $(1,75 \mathrm{~T})$.

Nous nous proposons d'exposer et de discuter les résultats obtenus à l'aide d'un perméamètre mis au point pour déterminer les propriétés magnétiques de rubans MG $2605 \mathrm{Co}$, bruts de trempe, en fonction de la traction exercée. Les caractéristiques présentées correspondent aux courbes $B(H)$ et aux pertes électromagnétiques, mesurées dans un domaine de fré- 
quences compris entre $50 \mathrm{~Hz}$ et $50 \mathrm{kHz}$ et pour des contraintes atteignant $75 \mathrm{daN} . \mathrm{mm}^{-2}(750 \mathrm{MPa})$. La composition des rubans testés est donnée par la formule $\mathrm{Fe}_{67} \mathrm{Co}_{18} \mathrm{~B}_{14} \mathrm{Si}_{1}$; le coefficient de magnétostriction $\lambda_{\mathrm{s}}$ vaut environ $35 \times 10^{-6}$. La résistance à la rupture est supérieure à $150 \mathrm{daN} . \mathrm{mm}^{-2}$. La limite élastique du matériau est mal connue; cependant, les observations montrent que tous les essais que nous avons réalisés sous traction correspondent au domaine des déformations élastiques.

\section{Dispositif expérimental.}

Le schéma du banc de mesure est précisé sur la figure 1. Il comprend un perméamètre à culasse équipé de deux bobines concentriques entourant l'échantillon à tester. L'enroulement primaire $b_{1}$, d'une longueur de $12 \mathrm{~cm}$ et constitué de 122 spires jointives, produit le champ magnétique. Le secondaire, $\mathrm{b}_{2}$, équidistant des extrémités de l'enroulement d'excitation, a une longueur de $1,5 \mathrm{~cm}$ et comporte $460 \mathrm{spi}$ res; il est destiné à la détermination du flux magnétique à travers le ruban. Les deux culasses symétriques $C_{1}$ et $C_{2}$, constituées de tôles à grains orientés $\mathrm{Fe}-\mathrm{Si}$ de $50 \mu \mathrm{m}$ d'épaisseur, assurent la fermeture du circuit magnétique ; leur section $\left(30 \mathrm{~cm}^{2}\right)$ a été choisie de manière à ce que la différence de potentiel magnétique aux bornes de l'éprouvette soit négligeable devant la force magnétomotrice nécessaire pour magnétiser l'échantillon.

L'enroulement primaire est alimenté par un oscil-

en cascade, à un amplificateur de puissance suivi d'un adaptateur d'impédance. Le courant, mesuré à l'aide d'une sonde à effet Hall, est proportionnel au champ magnétique $H$ à la surface du ruban. La f.é.m. induite aux bornes du secondaire est amplifiée puis intégrée; le signal délivré par l'intégrateur est proportionnel au flux à travers l'enroulement. Le flux, et donc l'induction magnétique moyenne, à travers le ruban sont obtenus en déduisant le flux à travers la couche d'air située entre le ruban et la bobine détectrice. Les signaux, images du champ et de l'induction magnétiques, sont appliqués respectivement sur les voies $X$ et $Y$ d'un oscilloscope à mémoire numérique. Un " bus " IEEE réalise l'interface entre l'oscilloscope et un microcalculateur. Les caractéristiques magnétiques du matériau sont déduites des cycles d'hysté-

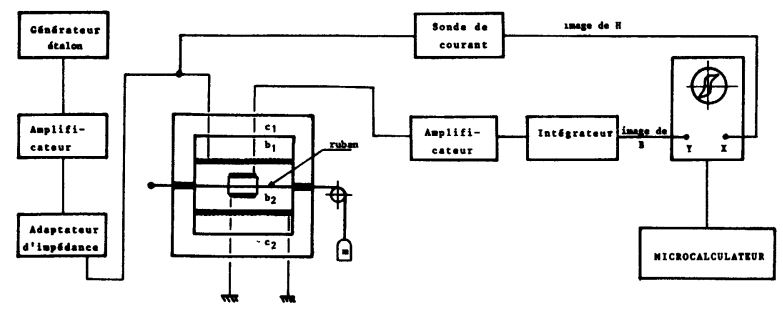

Fig. 1. - Dispositif expérimental.

[Schematic diagram of the magnetizing system.] résis. Le calculateur est programmé de manière à déterminer, pour chaque essai, les valeurs maximales du champ et de l'induction, la perméabilité relative et les pertes.

Le système de traction est dimensionné de manière à exercer, sur un ruban unitaire, des contraintes longitudinales jusqu'à la limite de rupture $(\sim 100$ daN. $\mathrm{mm}^{-2}$ ). Un entrefer, aménagé entre les deux culasses, permet au ruban de coulisser librement sur son axe. Les contraintes $\sigma$ sont calculées en effectuant le rapport entre l'effort exercé et la section du ruban, mesurée à l'aide d'un palpeur micrométrique.

Le dispositif permet de caractériser des échantillons de 10 à $50 \mathrm{~mm}$ de largeur et de 20 à $60 \mu \mathrm{m}$ d'épaisseur. Nous avons relevé les caractéristiques magnétiques de la culasse afin d'apprécier l'erreur commise en négligeant sa réluctance devant celle du ruban. Pour un échantillon de $50 \mathrm{~mm}$ de large et de perméabilité relative $\mu_{\mathrm{r}}=10^{5}$, le rapport des réluctances est, à $50 \mathrm{~Hz}$, d'environ $3 \%$. La valeur de ce rapport diminue en même temps que la perméabilité de l'amorphe testé. Il est plus difficile d'évaluer la réluctance de l'entrefer aménagé pour permettre au ruban de coulisser. Si l'on suppose que les lignes d'induction y sont uniformément réparties (hypothèse discutable), l'ordre de grandeur du rapport entre la réluctance de l'entrefer et celle de l'échantillon précédemment défini est encore de $3 \%$. Cette valeur est évidemment sous-estimée. Ainsi, l'hypothèse selon laquelle la différence de potentiel magnétique aux bornes de

motrice nécessaire pour magnétiser l'échantillon n'est effectivement vérifiée, à $1 \%$ près, que pour des rubans dont la perméabilité relative est inférieure à $2 \times 10^{4}$. Néanmoins, nous calculerons le champ à la surface du ruban en supposant que l'hypothèse précédente est toujours satisfaite. Par la suite, nous envisageons de modifier le perméamètre en utilisant un matériau mieux adapté pour la culasse (Mumétal par exemple) et en l'équipant d'un bobinage de compensation [11].

Les mesures sont réalisées en champ sinusoïdal pour des fréquences comprises entre $50 \mathrm{~Hz}$ et $50 \mathrm{kHz}$.

\section{Résultats.}

Les caractéristiques magnétiques sont relevées sur des rubans de $25 \mu \mathrm{m}$ d'épaisseur et de $10 \mathrm{~mm}$ de largeur. La dispersion est respectivement $\pm 1 \mu \mathrm{m}$ et $\pm 0,25 \mathrm{~mm}$.

Lors des essais sous contrainte, celle-ci est appliquée dans un ordre croissant, afin de ne pas introduire d'hystérésis mécanique.

Les courbes de la figure 2 représentent la variation de l'induction $B$ en fonction du champ appliqué $H$ (lieu des maximums des cycles d'hystérésis), pour les différentes fréquences imposées et en absence d'effort de $\operatorname{traction}(\sigma=0)$. Il apparaît que l'induction semble tendre vers une limite nettement inférieure à celle annoncée par le fabricant. Cette caractéristique est commune à la plupart des ferromagnétiques amorphes 


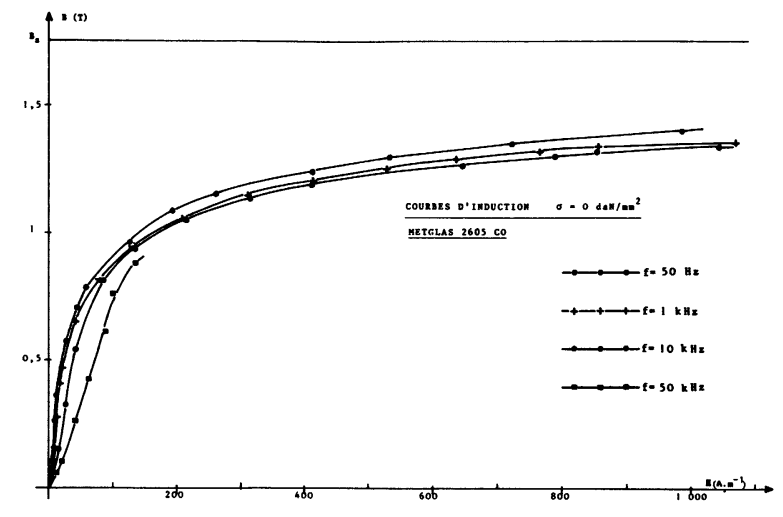

Fig. 2. - Courbes d'induction $B(H)$ en absence de contraintes appliquées.

[Flux density versus magnetic field without load.]

non recuits; leur structure magnétique est, en effet, constituée d'au moins deux types de domaines qui réagissent différemment à l'action d'une excitation magnétique [12]. On observe ainsi une pseudosaturation correspondant au réarrangement des domaines les plus larges. La saturation réelle du matériau n'est obtenue qu'avec des champs suffisamment intenses pour exciter les microdomaines enserrés dans les précédents. Par ailleurs, on constate que, pour $H$ imposé, l'induction décroît lorsque la fréquence augmente. Si l'on admet que la d.d.p. magnétique aux bornes de l'échantillon reste négligeable à haute fréquence, ce phénomène rend compte de l'effet de peau dont l'influence augmente avec la fréquence. Dans la méthode utilisée, la valeur du champ $H$ à la surface du ruban est fixée par le courant à travers le bobinage primaire; par contre, l'induction mesurée $B$ correspond à la valeur moyenne à travers la section de l'échantillon. Lorsque la fréquence augmente, l'exclusion des lignes d'induction entraîne une diminution de $B, H$ conservant la valeur imposée.

Les figures 3 et 4 représentent, pour une fréquence de $50 \mathrm{~Hz}$, l'évolution des courbes $B(H)$ en fonction de la traction exercée sur le ruban. On constate que, jusqu'à $10 \mathrm{daN} . \mathrm{mm}^{-2}$, la contrainte a pour effet, à excitation donnée, d'augmenter la valeur de l'induction (Fig. 3). En particulier, pour une contrainte de $10 \mathrm{daN} . \mathrm{mm}^{-2}$, l'induction atteinte $(1,7 \mathrm{~T})$ est proche de l'induction à saturation $(1,75 \mathrm{~T})$. Pour des tractions plus fortes, on note une diminution des caractéristiques qui restent supérieures à celle obtenue pour le ruban non contraint. L'évolution observée s'explique par une modification de la structure magnétique du matériau. Dans le cas des ferromagnétiques amorphes à coefficient de magnétostriction positif, les tractions tendent à organiser les domaines en bandes parallèles à l'axe de l'effort exercé [13]. Il s'ensuit, dans cette direction, une augmentation de la perméabilité. Cela est conforme aux résultats présentés sur la figure 3. Cette modification est comparable à celle induite par un traitement thermique sous champ longitudinal [4-6].

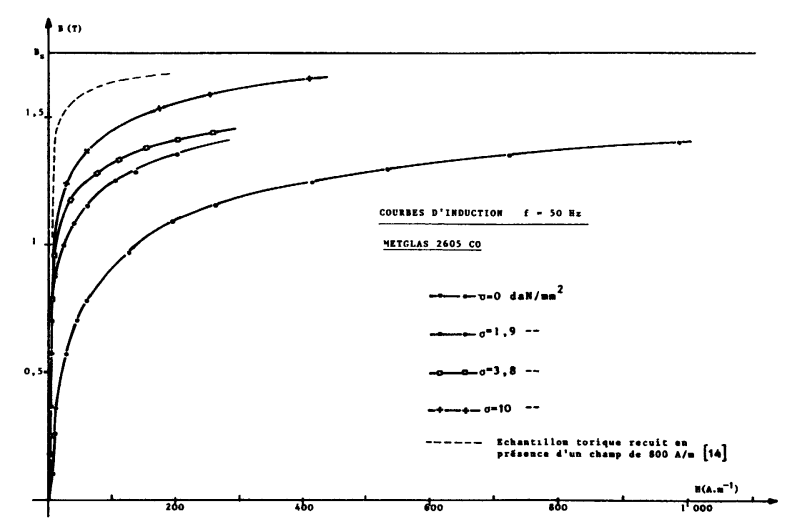

Fig. 3. - Courbes d'induction $B(H)$ du matériau contraint à $f=50 \mathrm{~Hz}$

[Flux density versus magnetic field at $f=50 \mathrm{~Hz}$ with various tensile stresses.]

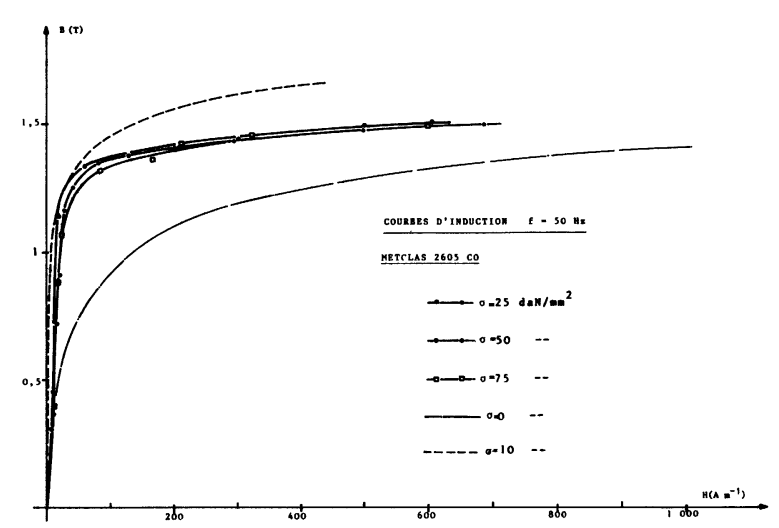

Fig. 4. - Courbes d'induction $B(H)$ à $f=50 \mathrm{~Hz}$.

[Flux density versus magnetic field at $f=50 \mathrm{~Hz}$ with various tensile stresses.]

A titre comparatif, nous avons porté, sur la figure 3, la courbe $B(H)$ relevée, à $50 \mathrm{~Hz}$, sur un tore recuit sous champ circonférentiel de $800 \mathrm{~A} \cdot \mathrm{m}^{-1}$ [14]. Les résultats suggèrent que, dans ce cas, l'organisation des domaines en bandes est plus complète.

Pour des contraintes supérieures à $10 \mathrm{daN} . \mathrm{mm}^{-2}$, on observe une diminution de l'induction lorsque l'effort de traction augmente. L'explication de ce phénomène est délicate et nécessiterait l'examen in situ de la structure magnétique. Cependant, il est vraisemblable que les efforts exercés ne se répartissent pas uniformément au sein du matériau. Il s'ensuit des contraintes de cisaillement qui augmentent avec la traction (et donc l'allongement du ruban) et dont l'action est contraire à celle des efforts longitudinaux. Ainsi, l'existence d'une contrainte appliquée optimale pour laquelle l'induction est maximale traduit l'action de deux phénomènes antagonistes.

Les résultats obtenus pour les fréquences de $1 \mathrm{kHz}$, $10 \mathrm{kHz}$ et $50 \mathrm{kHz}$ sont comparables à ceux présentés sur les figures 3 et 4 et ne seront donc pas décrits dans cet article. 
Les courbes des figures 5 et 6 traduisent l'évolution des pertes totales, calculées à partir de l'aire des cycles, en fonction de la traction exercée, pour deux valeurs de l'induction $(0,5$ et $1 \mathrm{~T})$. On note une évolution différente selon la fréquence : à $50 \mathrm{~Hz}$, les pertes diminuent, passent par un minimum puis augmentent avec la contrainte; à plus haute fréquence $(1 \mathrm{kHz}$ et $10 \mathrm{kHz}$ ), on observe que le minimum est moins prononcé; enfin à $50 \mathrm{kHz}$, les pertes augmentent de façon monotone avec la traction. En faisant l'hypothèse que les pertes totales sont en majeure partie des pertes par hystérésis à basse fréquence et des pertes par courants de Foucault à haute fréquence, le comportement observé peut encore s'expliquer par des modifications de la structure magnétique induites par les efforts de traction. En effet, une structure en bandes parallèles conduit à de faibles champs coercitifs et donc à de faibles pertes par hystérésis; par contre, les pertes par courants de Foucault ont tendance à augmenter avec la taille des domaines. Ainsi, les observations, effectuées pour des contraintes inférieures à $10 \mathrm{daN} \cdot \mathrm{mm}^{-2}$, rendraient compte d'une organisation des domaines en bandes parallèles à l'axe de l'effort exercé. Dans le cas des fortes contraintes, toute explication devient hasardeuse; l'interprétation nécessite alors la détermination de paramètres complémentaires afin de préciser la structure magnétique du matériau.

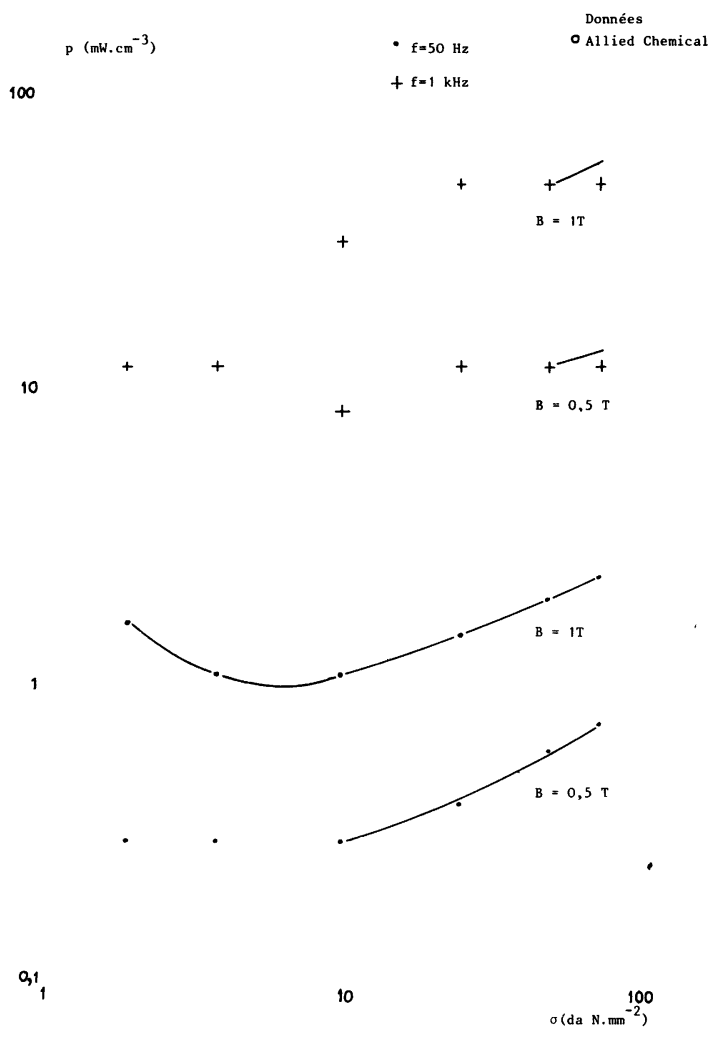

Fig. 5. - Pertes totales en fonction de la contrainte exercée $(f=50 \mathrm{~Hz}$ et $f=1 \mathrm{kHz})$.

[Power loss versus applied tensile stress at $f=50 \mathrm{~Hz}$ and $1 \mathrm{kHz}$.]

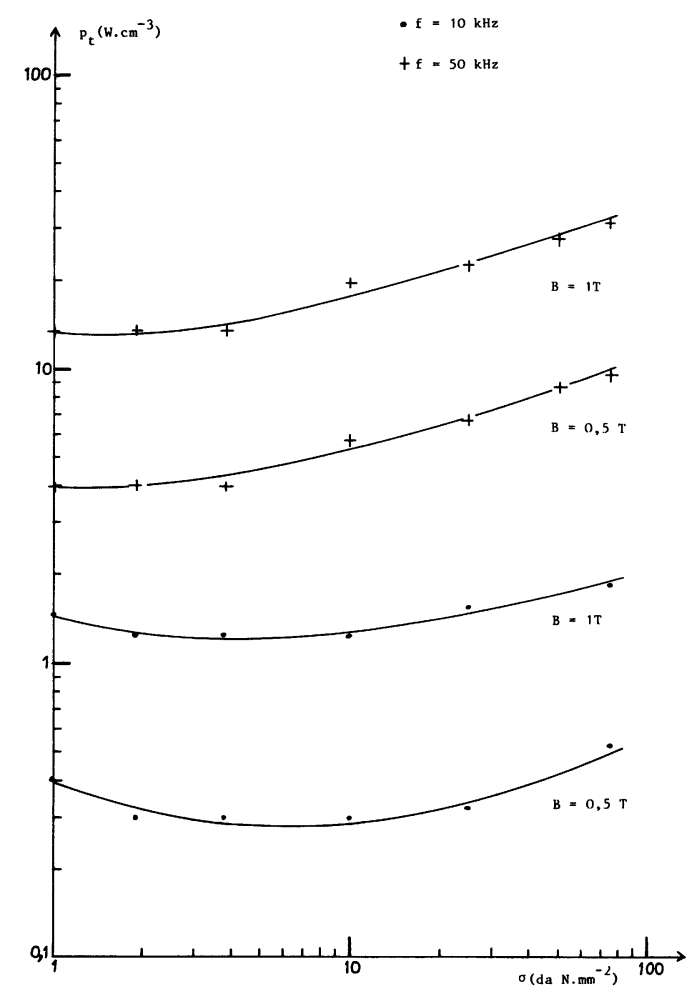

Fig. 6. - Pertes totales en fonction de la contrainte exercée $(f=10 \mathrm{kHz}$ et $f=50 \mathrm{kHz})$.

[Power loss versus applied tensile stress at $f=10 \mathrm{kHz}$ and $50 \mathrm{kHz}$.]

Nous avons porté, sur la figure 5, les valeúrs des pertes associées à un tore recuit sous champ, pour une induction de $1 \mathrm{~T}$ et des fréquences de 50 et $1000 \mathrm{~Hz}$ (Allied Chemical). Il est intéressant de noter que la valeur indiquée à $50 \mathrm{~Hz}$ est inférieure à celle que nous obtenons en l'absence de contrainte, tandis qu'à $1000 \mathrm{~Hz}$ elle est supérieure. Cela peut s'expliquer en considérant que le recuit sous champ induit une anisotropie uniaxiale et s'accompagne d'une diminution des pertes par hystérésis et d'une augmentation des pertes par courants de Foucault. Ces dernières peuvent être réduites à l'aide de traitements thermiques plus élaborés, par exemple un recuit sous champ oblique $[9,10]$.

L'influence des contraintes de traction sur les pertes dissipées dans des rubans de composition différente, recuits ou non, est analogue à celle que nous observons $[7,8,15,16]$. Le domaine de fréquence exploré est plus limité $(f<1000 \mathrm{~Hz})$. Par contre, les travaux cités en référence montrent l'action des traitements thermiques sur les pertes en fonction de l'anisotropie induite. Actuellement, nous poursuivons notre étude dans cette direction.

\section{Conclusion.}

Du point de vue de l'utilisateur, l'action d'un effort de traction appliqué parallèlement au champ magnétique est positive si on se limite à des fréquences de $10 \mathrm{kHz}$. 
Cependant, dans un rotor de machine électrique, il existe certaines zones où les lignes d'induction sont perpendiculaires à la force centrifuge. Il est vraisemblable que, dans ce cas, les évolutions des caractéristiques magnétiques diffèrent de celles présentées ici. Pour caractériser l'effet des contraintes transversales, il est nécessaire de disposer de rubans plus larges que ceux qui sont actuellement commercialisés. Une telle méthode devrait, pourtant, bientôt être possible. $\mathrm{La}$ structure du rotor sera fonction de ces résultats. Par ailleurs, il faut préciser que du fait de leur faible épaisseur, l'état de surface des rubans amorphes est un paramètre qui influence fortement les caractéristiques magnétiques. Il s'ensuit des écarts selon l'épaisseur et la largeur des échantillons $[17,18]$. L'utilisation de rubans de grande largeur peut s'accompagner d'une diminution des performances par rapport à celle de rubans étroits.

Les ferromagnétiques amorphes se caractérisent par des pertes par courants de Foucault importantes. Un des problèmes actuels est de définir un traitement susceptible de les diminuer tout en laissant intactes les caractéristiques mécaniques. Il est également important de caractériser l'évolution du matériau au cours du temps lorsqu'il est soumis à des contraintes thermiques qui, dans le cas de machines à haute densité d'énergie, peuvent atteindre $200^{\circ} \mathrm{C}$.

D'un point de vue plus fondamental, nous avons défini un programme d'essais destiné à étudier l'effet des contraintes appliquées en fonction du traitement thermique (recuit sans champ destiné à relaxer les contraintes internes, recuit sous champ et/ou sous contrainte longitudinale susceptible de créer une anisotropie uniaxiale). Dans le but de mieux définir l'action des efforts exercés, nous mettons au point un système permettant le relevé des cycles d'hystérésis en régime pseudo-continu. De même, nous cherchons à visualiser la structure magnétique afin de mieux comprendre les évolutions observées, en particulier dans le domaine des fortes contraintes. Enfin, l'extension de cette étude à des rubans dont le coefficient de magnétostriction est différent doit permettre de préciser l'influence de ce paramètre sur les propriétés du matériau.

\section{Bibliographie}

[1] Egami, T., Flanders, P. J., Graham, C. D. Jr., Appl. Phys. Lett. 26 (1975) 128.

[2] O’Handley, R. C., Solid State Commun. 21 (1977) 1119.

[3] Ito, S., Aso, K., Makino, Y., Uedaira, S., Appl. Phys. Lett. 37 (1980) 665.

[4] Egami, T., Flanders, P. J., AIP Conf. Proc. 29 (1976) 220.

[5] Fujimori, H., Morita, H., Oвi, Y., OHto, S., Amorphous Magnetism II (Plenum Press, N.Y.) 1977, p. 393.

[6] Luborsky, F. E., WALTER, J. L., IEEE Trans. Magn. MAG 13 (1977) 953.

[7] Nielsen, O. V., Nielsen, H. J. V., J. Magn. Mat. 22 (1980) 21.

[8] Nielsen, H. J. V., Gynther Nielsen, K., Nielsen, O. V., IEEE Trans. Magn. MAG 18 (1982) 904.

[9] Fujimori, H., Yoshimoto, H., Masumoto, T., Mitera, T., J. Appl. Phys. 52 (1981) 1893.
[10] Luborsky, F. E., Livingston, J. D., IEEE Trans. Magn. MAG 18 (1982) 908.

[11] ElnéKavé, N., IEEE Trans. Magn. (1969) 662.

[12] Kronmüller, H., J. Physique Colloq. 41 (1980) C8-618.

[13] Dong, X. Z., Kronmüller, H., Phys. Status Solidi (a) 70 (1982) 451.

[14] TAHIR, Contribution à l'étude de l'influence de traitements magnétothermiques sur les propriétés de divers rubans ferromagnétiques amorphes, Mémoire CNAM Paris (1983).

[15] Blundell, M. G., Graham, C. D. Jr., Overshott, K. J., J. Magn. Magn. Mat. 19 (1980) 174.

[16] Overshott, K. J., Blundell, M. G., IEEE Trans. Magn. MAG 16 (1980) 1221.

[17] Blundell, M. G., Overshott, K. J., IEEE Trans. Magn. MAG 16 (1980) 1224.

[18] WANG, Y. J., Kronmüller, H., Phys. Status Solidi (a) 70 (1982) 415. 